\title{
Anticompetition of laser modes
}

\author{
Ching-Fuh Lin, ${ }^{\text {a) }}$ Chi-Chia Huang, Fei-Hung Chu, and Yi-Shin Su \\ Graduate Institute of Electro-Optical Engineering, National Taiwan University, Taipei 106, Taiwan, \\ Republic of China
}

(Received 13 January 2003; accepted 4 April 2003)

\begin{abstract}
Anticompetition of laser modes is observed. In this behavior, the increase of intensity in one lasing mode could enhance the intensity of another mode, which is opposite to the usual competition behavior and so-called anticompetition. In our experiments using the semiconductor laser with very broadband gain medium, anticompetition exists when the laser modes have their wavelengths widely separated. Anticompetition can be observed for spectral separation of $138 \mathrm{~nm}$ and is even more prominent for spectral separation up to $167 \mathrm{~nm}$. Theoretical analysis shows that anticompetition is due to the physics similar to optical pumping. (C) 2003 American Institute of Physics. [DOI: 10.1063/1.1578708]
\end{abstract}

Mode competition is a common phenomenon in lasers. Soon after the invention of lasers, ${ }^{1,2}$ competition of laser modes was noticed and analyzed. ${ }^{3}$ Because the total gain of a laser medium is constant under a fixed pumping level, the modes under oscillation compete for the total gain. As a result, when one mode increases its intensity, it suppresses the other modes. The competition could lead to phenomena such as bistability, ${ }^{4,5}$ tristability, ${ }^{6,7}$ and even chaotic behaviors, ${ }^{8,9}$ which were found useful for optical logic, optical switching, data encryption, etc. ${ }^{10,11}$ Competition dynamics is also involved in injection locking, ${ }^{12}$ mode locking, ${ }^{13-15}$ and crossgain modulation in optical amplifiers. ${ }^{16}$ For decades, competition has been thought to be inevitable among laser modes. However, we discover that laser modes could behave in a way opposite to the competition. In our discovery, the increase of intensity in one lasing mode was found to enhance the intensity of another mode. We call such behavior as anticompetition of laser modes.

The anticompetition of laser modes is explored using the laser system schematically shown in Fig. 1. The system has a reflected-type grating telescope configuration of external cavity. The gain medium used in the cavity is a bent-stripe ridge waveguide semiconductor optical amplifier (SOA). ${ }^{17}$ The waveguide has its right-hand side normal to the cleaved facet of the SOA, while its left-hand side is bent and oriented at an angle of $7^{\circ}$ to the normal of another cleaved facet. Nonidentical multiple quantum wells (MQWs) are used in the SOA to provide a very broad gain bandwidth. ${ }^{18}$ Past experiments showed that such broadband gain could be used to support two modes with spectral separation tunable from a few to $170 \mathrm{~nm} .{ }^{19}$ Two collimators are used to collect light emitted from the SOA. The laser cavity is formed between the right-hand facet of the SOA and the mirrors A and $\mathrm{B}$. The grating in the cavity is Au-coated and has 600 lines $/ \mathrm{mm}$. It is used to disperse the light of different wavelengths. A lens with a focal length of $10 \mathrm{~cm}$ was placed at the distance of 10 $\mathrm{cm}$ from both the grating and mirrors A and $\mathrm{B}$. According to the grating principle, the beams of different wavelengths are

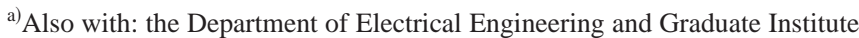
of Electronics Engineering; electronic mail: cflin@cc.ee.ntu.edu.tw
}

horizontally dispersed at the mirrors with a spatial separation $W \sim L(1 / \Lambda) 1 / \cos \theta_{R} \Delta \lambda$, where $\Delta \lambda$ is the spectral separation, $\Lambda$ is the grating period, $\theta_{R}$ is the first-order diffraction angle, and $L$ is the distance between the lens and the grating. Two modes are selected using double slits in front of mirrors A and $\mathrm{B}$ for the competition experiment. The separation of the two slits is adjustable so that the spectral separation of the two modes can be varied. The output light is divided into two beams by a beam splitter. One beam is delivered into a monochromator for spectrum analysis. The other beam is dispersed by another grating for the measurement of the intensities at two wavelengths.

A neutral-density (ND) filter with variable loss is inserted in either path of the two modes so that the intensity of one mode can be controlled. If competition exists between the two modes, increasing the intensity of one mode leads to the decreased intensity of another mode and vice versa for anticompetition. The phenomenon of anticompetition is observed when the two modes are widely separated.

Figure 2 shows the lasing spectra of the two modes with anticompetition. The wavelengths of the two modes are

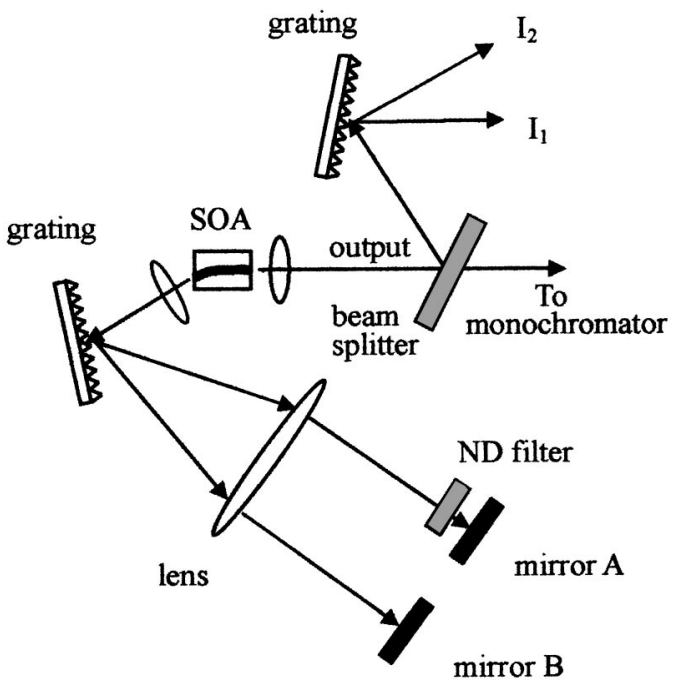

FIG. 1. A schematic of the laser system for the experiment of anticompetition between laser modes. The system has the reflected-type grating telescope configuration of external cavity. 


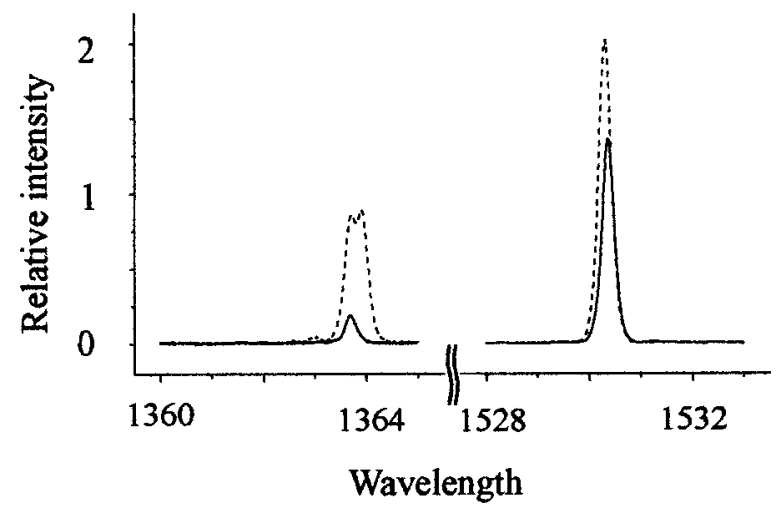

FIG. 2. The lasing spectra of the two modes with anticompetition. The solid line shows the spectrum for the short-wavelength path with large loss, controlled by the ND filter. The short-wavelength and the long-wavelength modes both have low intensities. The dashed line shows the spectrum for the short-wavelength path with small loss, controlled by the ND filter. The short-wavelength and the long-wavelength modes both have increased intensities.

around 1363.5 and $1530.5 \mathrm{~nm}$, respectively. The current injected into the SOA is $146 \mathrm{~mA}$ and the temperature is controlled at $22.7^{\circ} \mathrm{C}$. In these measurements, the ND filter is inserted in the optical path of the short-wavelength mode. Therefore, the intensity of the 1363.5 -nm mode is controlled, while the intensity of the 1530.5 -nm mode varies accordingly. The solid and dashed lines show the spectra for the 1363.5-nm mode controlled at small and large intensities, respectively. As the intensity of the 1363.5 -nm mode is controlled to be small, the intensity of the $1530.5-\mathrm{nm}$ mode is also small. On the other hand, if the intensity of the $1363.5-\mathrm{nm}$ mode is large, the intensity of the $1530.5-\mathrm{nm}$ mode increases, too. This phenomenon is opposite to the usual behavior of mode competition. To clearly demonstrate the conditions of anticompetition, the variation of the intensity of $1530.5-\mathrm{nm}$ mode $\left(I_{2}\right)$ with the intensity of $1363.5-\mathrm{nm}$ mode $\left(I_{1}\right)$ is measured, and is shown in Fig. 3(a). It is obvious that $I_{2}$ increases with $I_{1}$ as the intensity of $I_{1}$ increases from zero to $0.35 \mathrm{~mW}$.

The two modes with reduced spectral separation are also studied. Under the same injection current and the same temperature, as the spacing of the double slits is reduced, the laser shown in Fig. 1 could oscillate at two other wavelengths: $1388 \mathrm{~nm}\left(I_{1}\right)$ and $1526 \mathrm{~nm}\left(I_{2}\right)$. Again, the variable ND filter is placed in the optical path of the short-wavelength mode to control the $I_{1}$ intensity. The anticompetition still exists, but only for a small regime of $I_{1}$ values. When $I_{1}$ is larger than $0.25 \mathrm{~mW}$, the two modes have usual competition. The corresponding $I_{2}$ versus $I_{1}$ curve is shown in Fig. 3(b). If the spectral separation is further reduced, only competition exists. Figure 3(c) shows $I_{2}$ versus $I_{1}$ curve for this situation with wavelengths selected at 1378 and $1413 \mathrm{~nm}$.

Previous models of competition usually use the following rate equations to describe the gain saturation: ${ }^{7,20}$

$$
\begin{aligned}
& \frac{d I_{1}}{d t}=\left(\frac{g_{10}}{1+S_{1} I_{1}+C_{12} I_{2}}-l_{1}\right) I_{1}, \\
& \frac{d I_{2}}{d t}=\left(\frac{g_{20}}{1+S_{2} I_{2}+C_{21} I_{1}}-l_{2}\right) I_{2},
\end{aligned}
$$
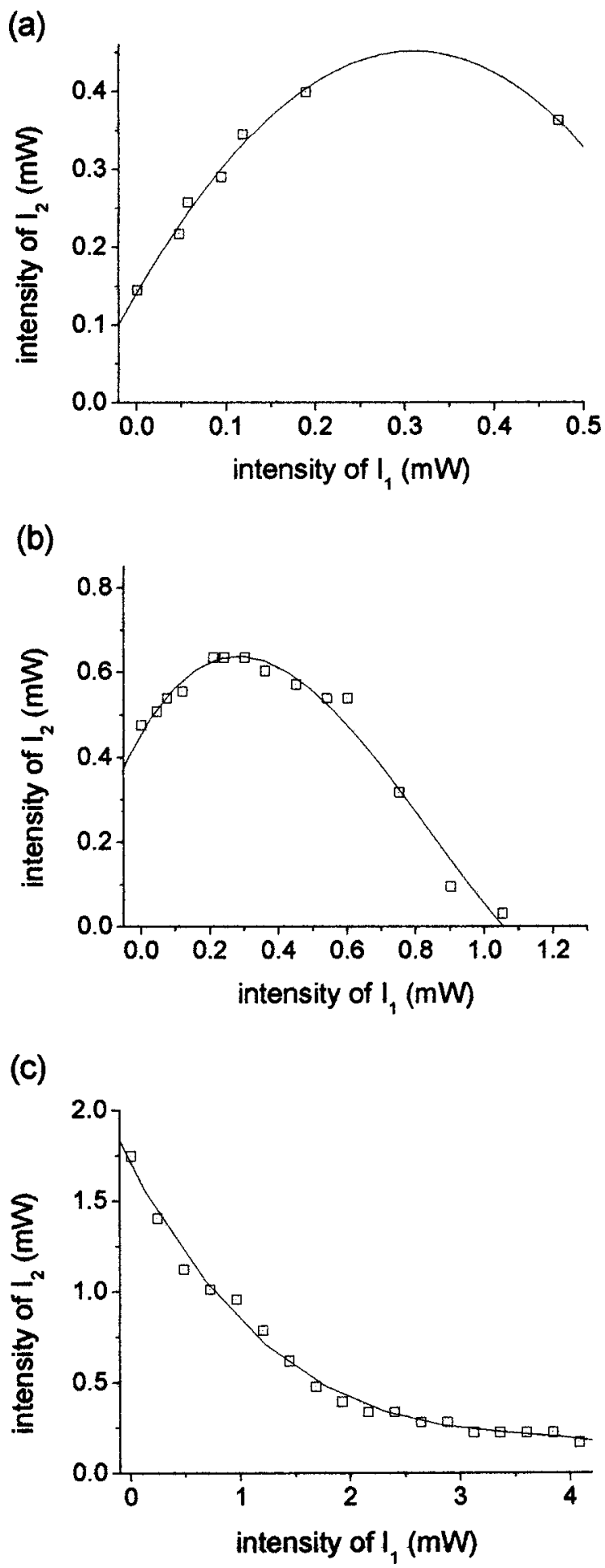

FIG. 3. Variation of $I_{2}$ with $I_{1}$. The intensity of $I_{1}$ is controlled by the loss of the ND filter inserted in this optical path, while the intensity of $I_{2}$ changes accordingly. (a) wavelength of $I_{1}=1363.5 \mathrm{~nm}$, wavelength of $I_{2}$ $=1530.5 \mathrm{~nm}$; (b) wavelength of $I_{1}=1388 \mathrm{~nm}$, wavelength of $I_{2}$ $=1526 \mathrm{~nm}$, and (c) wavelength of $I_{1}=1413 \mathrm{~nm}$, wavelength of $I_{2}$ $=1378 \mathrm{~nm}$. Square dots: experimental data; solid line: fitted curve.

where the first term in parentheses [Eq. (1a)] represents the gain with self- and cross-saturation; $g_{10}$ and $g_{20}$ are the unsaturated gains of the modes $I_{1}$ and $I_{2}$, respectively; $l_{1}$ and $l_{2}$ are losses of the modes $I_{1}$ and $I_{2}$, respectively. In reality, the denominators in Eqs. (1a) and (1b) are not linear functions of $I_{1}$ and $I_{2} \cdot{ }^{20}$ The rate equations can be put into more general forms: 


$$
\begin{aligned}
& \frac{d I_{1}}{d t}=\left[G_{1}\left(I_{1}, I_{2}\right)-l_{1}\right] I_{1}, \\
& \frac{d I_{2}}{d t}=\left[G_{2}\left(I_{1}, I_{2}\right)-l_{2}\right] I_{2},
\end{aligned}
$$

with $G_{1}\left(I_{1}, I_{2}\right)$ and $G_{2}\left(I_{1}, I_{2}\right)$ representing general relations of the gains and the intensities $I_{1}$ and $I_{2}$. In the steady state, $d I_{1} / d t=d I_{2} / d t=0$. Equations (2a) and (2b) can then be written as

$$
\begin{aligned}
& {\left[G_{1}\left(I_{1}, I_{2}\right)-l_{1}\right] I_{1}=0,} \\
& {\left[G_{2}\left(I_{1}, I_{2}\right)-l_{2}\right] I_{2}=0,}
\end{aligned}
$$

where $G_{1}\left(I_{1}, I_{2}\right)-l_{1}=0$ and $G_{2}\left(I_{1}, I_{2}\right)-l_{2}=0$ represent two curves on the $I_{1}-I_{2}$ phase plane.

Equations (3a) and (3b) have three nontrivial steadystate solutions: (i) $I_{1}=0$ and $G_{2}\left(I_{1}, I_{2}\right)-l_{2}=0$, (ii) $G_{1}\left(I_{1}, I_{2}\right)-l_{1}=0$ and $I_{2}=0$, and (iii) $G_{1}\left(I_{1}, I_{2}\right)-l_{1}=0$ and $G_{2}\left(I_{1}, I_{2}\right)-l_{2}=0$. The solutions of the first two cases are on the axes of the $I_{1}-I_{2}$ phase plane, so either $I_{1}$ or $I_{2}$ is zero. For the third case, the intersection of the two curves gives the intensities of the two modes, $I_{1}$ and $I_{2}$, which both are not zero. Although the exact mathematical forms of $G_{1}\left(I_{1}, I_{2}\right)$ and $G_{2}\left(I_{1}, I_{2}\right)$ are generally not known, they can be determined by experiment. With the loss $l_{1}$ varied, the equation $G_{1}\left(I_{1}, I_{2}\right)-l_{1}=0$ gives a family of curves. The intersection of this family of curves with the curve $G_{2}\left(I_{1}, I_{2}\right)-l_{2}=0$ (with $l_{2}$ fixed) then traces out part of the curve $G_{2}\left(I_{1}, I_{2}\right)-l_{2}=0$. Similarly, with the loss $l_{2}$ varied, the curve $G_{1}\left(I_{1}, I_{2}\right)-l_{1}=0$ (with $l_{1}$ fixed) can be partially traced out. Therefore, Figs. 3(a) -3 (c) represent the gain function $G_{2}\left(I_{1}, I_{2}\right)-l_{2}=0$ for three spectral separations.

The saturation effect causes the gain to decrease with the intensity, so the self-saturation leads to $\partial G_{2} / \partial I_{2}<0$. The competition is due to cross-saturation, ${ }^{4,5,20}$ so $\partial G_{2} / \partial I_{1}<0$. Thus, for competition situation, the gain curve $G_{2}\left(I_{1}, I_{2}\right)$ $-l_{2}=0$ should have the slope $d I_{1} / d I_{2}<0$, which is the case for Fig. 3(c) and for previous experiments. ${ }^{5,8}$ For the case of anticompetition shown in Figs. 3(a) and 3(b), $d I_{1} / d I_{2}>0$. Because self-saturation still gives $\partial G_{2} / \partial I_{2}<0$, it must be that $\partial G_{2} / \partial I_{1}>0$. As a result, the power-series expansion of $G_{2}\left(I_{1}, I_{2}\right)$ consists of a term $\left(\partial G_{2} / \partial I_{1}\right) I_{1}\left(=\gamma I_{1}>0\right)$, which means the condition like optical pumping. That is, the shortwavelength mode gives away its optical power to the longwavelength mode. However, the two-mode operation is not exactly the same as optical pumping. As shown in Figs. 3(a) and 3(b), some regime of competition still exists, indicating that the cross-saturation plays an important role again for certain intensity of $I_{1}$.
The experiments have also been done with the ND filter placed in the path of the long-wavelength mode. No anticompetition is observed for this situation, whether the spectral separation is large or small. It shows that the longwavelength mode, having a lower energy than the shortwavelength mode, cannot provide a function like optical pumping.

In conclusion, anticompetition of laser modes is discovered. The phenomenon of anticompetition exists when the laser modes have their wavelengths widely separated. In our experiments using the semiconductor laser with nonidentical MQWs anticompetition can be observed for spectral separation of $138 \mathrm{~nm}$. The anticompetition is even more prominent for spectral separation up to $167 \mathrm{~nm}$. Theoretical analysis shows that anticompetition is due to the physics similar to optical pumping.

This work is supported in part by National Science Council, Taipei, Taiwan, R.O.C. under the contract No. NSC91-2215-E-002-025.

${ }^{1}$ T. H. Maiman, Nature (London) 6, 106 (1960).

${ }^{2}$ A. Javan, W. R. Bennett, Jr., and D. R. Herriot, Phys. Rev. Lett. 6, 106 (1961).

${ }^{3}$ W. E. Lamb, Phys. Rev. A 134, 1429 (1964).

${ }^{4}$ K. Shimoda, Introduction to Laser Physics (Springer, Berlin, 1984), p. 187.

${ }^{5}$ A. E. Siegman, Lasers (University Science Books, 1986).

${ }^{6}$ M. Watanabe, H. Itoh, S. Mukai, and H. Yajima, Appl. Phys. Lett. 50, 427 (1987).

${ }^{7}$ C.-F. Lin and P.-C. Ku, IEEE J. Quantum Electron. 32, 1377 (1996).

${ }^{8} \mathrm{H}$. Kawaguchi, Bistability and Nonlinearities in Laser Diodes (Artech House, Norwood, MA, 1994), p. 199.

${ }^{9}$ S. Sivaprakasam, P. S. Spencer, P. Rees, and K. A. Shore, IEEE J. Quantum Electron. 38, 1155 (2002).

${ }^{10}$ H. Kawaguchi and I. S. Hidayat, Electron. Lett. 31, 1150 (1995).

${ }^{11}$ G. D. VanWiggeren and R. Roy, Science (Washington, DC, U.S.) 279, 1198 (1998).

${ }^{12}$ X. Jin and S. L. Chuang, Appl. Phys. Lett. 77, 1250 (2000).

${ }^{13}$ H. Statz and C. L. Tang, J. Appl. Phys. 36, 3923 (1965).

${ }^{14}$ L. E. Hargrove, R. L. Fork, and M. A. Pollack, Appl. Phys. Lett. 5, 4 (1964).

${ }^{15}$ P. W. Smith, Proc. IEEE 58, 1342 (1970).

${ }^{16}$ I. White, R. Penty, M. Webster, Y. J. Chai, A. Wonfor, and Sadegh Shahkooh, IEEE Communications Magazine 40, 74 (2002).

${ }^{17}$ C.-F. Lin and C.-S. Juang, IEEE Photonics Technol. Lett. 8, 206 (1996).

${ }^{18}$ C.-F. Lin, B.-R. Wu, L.-W. Laih, and T.-T. Shih, Opt. Lett. 26, 1099 (2001).

${ }^{19}$ C.-H. Chen, C.-F. Lin, Y.-S. Su, C.-C. Huang, and B.-R. Wu, CLEO2002, Long Beach, CA, 2002, Paper CWK.

${ }^{20}$ M. Sargent, III, M. O. Scully, and W. E. Lamb, Jr., Laser Physics (Addison-Wesley, Reading, MA 1974), p. 115. 
Applied Physics Letters is copyrighted by the American Institute of Physics (AIP). Redistribution of journal material is subject to the AIP online journal license and/or AIP copyright. For more information, see http:/ojps.aip.org/aplo/aplcr.jsp

Copyright of Applied Physics Letters is the property of American Institute of Physics and its content may not be copied or emailed to multiple sites or posted to a listserv without the copyright holder's express written permission. However, users may print, download, or email articles for individual use. 